\title{
RESONANT-TUNNELING DEVICES FOR MILLIMETER-WAVE GENERATION
}

\author{
R. K. Mains, I. Mehdi, and G. I. Haddad \\ Center for High-Frequency Microelectronics \\ Department of Electrical Engineering and \\ Computer Science \\ The University of Michigan \\ Ann Arbor, Michigan 48109
}

Received January 25, 1989

\begin{abstract}
Oscillations from resonant-tunneling diodes have been observed up to $200 \mathrm{GHz}$, and theoretical estimates predict that device performance should extend into the $\mathrm{THz}$ range. This paper addresses the issue of the ultimate frequency response and power generation capability of these devices. Techniques recently developed to solve the time-dependent Schrödinger equation are used to predict the rf power vs. frequency obtainable from resonant-tunneling diode oscillators, based on the calculated small-signal response. Factors limiting the rf power generation are considered, and methods for optimizing $\mathrm{rf}$ power output from these devices are presented. Also, recently obtained dc experimental results for the $\mathrm{In}_{.53} \mathrm{Ga}_{.47} \mathrm{As}-\mathrm{In}_{x} \mathrm{Al}_{1-x} \mathrm{As}$ heterostructure material system grown on InP are presented. Using a quasi-static approximation, the rf power available from these devices under large-signal conditions is estimated.
\end{abstract}




\section{Introduction}

Several etimates of the upper frequency limit of resonant-tunneling diode oscillators have been published [1]-[4], based upon estimates of the tunneling time combined with equivalent circuit models to include capacitive charging effects. Some estimates indicate that the intrinsic cutoff frequency extends into the $\mathrm{THz}$ range, however displacement current and circuit impedance limitations may reduce the maximum oscillation frequency to several hundred $\mathrm{GHz}$. Quantum transport calculations based on the Wigner function [5][8] and solution of the Schrödinger equation [9] have also demonstrated that resonant-tunneling devices are intrinsically capable of oscillating above $1 \mathrm{THz}$. Experimentally, oscillations up to $200 \mathrm{GHz}$ have been observed [10]-[11] although at disappointing power levels, with only a fraction of a microwatt obtained at $200 \mathrm{GHz}$. Also, experimental detection of the current response at $2.5 \mathrm{THz}$ for a GaAs-GaAlAs diode indicates that the device is still behaving quasistatically at that frequency [12].

Since development of resonant-tunneling diodes is still an active field of research, it is desirable to theoretically predict the ultimate power generating capabilities of these devices and to identify the device parameters important in optimizing performance. In this paper, two approaches are taken to estimate power generation. The results of Section 2 are based on small-signal solution of the Schrödinger equation. In Section 3, large-signal analyses are carried out based on the assumption that the device quasi-statically follows the I-V curve. Also, a comparison between rf power estimates based on theoretical and experimental I-V curves for a particular structure is presented. 


\section{Power Estimates and Device Opti- mization based on Small-Signal Cal- culations}

Techniques have been developed to obtain the small-signal response of resonant-tunneling diodes from solution of the time-dependent Schrödinger equation [9]. By this method the small-signal complex admittance of the device as a function of frequency is calculated. By assuming that at each frequency the device admittance remains constant over a finite range of rf voltage, estimates of $\mathrm{rf}$ power generation vs. frequency are obtained. The advantage of this method is that non-stationary behavior is taken into account, i.e. it is not necessary to assume that the device quasi-statically follows the dc I-V curve. However, this method has so far only been implemented for the case without inelastic scattering, and self-consistency has not yet been included. Therefore the device equivalent circuit must be augmented by an estimated device capacitance to account for charging currents.

All the calculations performed in this paper were at room temperature. Figure 1a shows the dc I-V curve calculated for a device consisting of two $28 \AA, .25 \mathrm{eV} \quad \mathrm{Al}_{.3} \mathrm{Ga}_{.7} \mathrm{As}$ barriers surrounding a $44.9 \AA \mathrm{GaAs}$ well, with $\mathrm{n}^{+} \mathrm{GaAs}$ contact regions doped at $10^{18} \mathrm{~cm}^{-3}$; Fig. $1 \mathrm{~b}$ shows the dc solution for this device at $\mathrm{V}_{d c}=.125 \mathrm{~V}$, corresponding to the peak current point in 1a. This device was biased at $\mathrm{V}_{d c}=.1615 \mathrm{~V}$, in the center of the negative differential conductance region, and the small-signal admittance about this $\mathrm{dc}$ point was calculated and is shown in Fig. 2a.

In Fig. 2a, the curves labeled $\operatorname{Re}\{Y\}$ and $\operatorname{Im}\{Y\}$ were calculated from the small-signal Schrödinger equation using the methods of Ref. [9]. Note that below approximately $200 \mathrm{GHz}$, the device negative conductance is nearly constant and is given by the negative slope of the dc I-V curve. Even at $1 \mathrm{THz}$, the negative conductance is over half its low-frequency value. This observation provides justification for the method wherein it is assumed that the device quasi-statically follows the dc I-V curve. However, it should be kept in mind that for structures with larger barriers, the small-signal neg- 
ative conductance rolls off at lower frequencies. The $\operatorname{Im}\{Y\}$ curve is a negative or inductive susceptance component. This is expected since the electron motion responds inductively to applied fields $[6,9]$. The $\omega \mathrm{C}$ component in Fig. $2 \mathrm{~b}$ was not obtained from the smallsignal analysis but was added to account for displacement currents. The per-unit area capacitance is given by:

$C=\frac{\epsilon}{D}$.

The depletion width D used to calculate $C$ was $101 \AA$, just the distance between the outer edge of the barriers. If self-consistency is included the depletion layer is wider than this value, and the power estimates become higher.

Since the data of Fig. 2a is per-unit area, the device area must be selected to calculate rf power. Also, the magnitude of the rf voltage across the device must be determined. To find the area, we assume that the device is matched to a circuit with resistance $R_{L} \Omega$. In that case, the device area must be :

$A=\frac{-G}{R_{L}} \frac{1}{G^{2}+(B+\omega C)^{2}}$,

where the susceptance in Eq. 2 has been divided into the (inductive) susceptance B calculated from the small-signal analysis in Fig. 2a and the capacitive component $\omega \mathrm{C}$ added to account for displacement currents. From Eq. 2, it is seen that the device area becomes larger as $R_{L}$ is reduced, and since the power scales with device area it is desirable to reduce the circuit resistance as much as possible. However there is a lower limit since eventually parisitic resistances become comparable to the desired circuit resistance and not all the rf power reaches the load resistance. In the calculations presented here, it is assumed that the minimum achievable circuit resistance is $1 \Omega$, so that the device area will be given by Eq. 2 with $R_{L}=1$. Note also from Eq. 2 that it is desirable to minimize the device per-unit area capacitance.

Having determined the device area, the $\mathrm{rf}$ power is given by:

$P_{r f}=\frac{V_{r f}^{2}}{2} \frac{1}{1+\left(\frac{B+\omega C}{G}\right)^{2}}$, 
where $R_{L}$ is assumed to be $1 \Omega$ and $V_{r f}$ is the peak rf voltage. The device efficiency is given by:

$\eta=\frac{P_{r f}}{P_{d c}}=-\frac{V_{r f}^{2} G}{2 V_{d c} J_{d c}}$.

The rf voltage appearing in Eqns. 3 and 4 is obtained as follows. A tangent line is drawn along the dc I-V curve at the dc operating point. The locations are found where this tangent line reaches the maximum and minimum current values on the I-V curve. One half the voltage separation between these two points is taken to be $V_{r f}$. For this case, $V_{r f}=14.0 \mathrm{mV}$ was used.

Fig. $2 \mathrm{~b}$ shows the results of the rf power calculations just described (solid curve), as well as the device area needed for $1 \Omega$ circuit matching (dashed curve). It is seen that over $90 \mu \mathrm{W}$ is expected from this device up to $400 \mathrm{GHz}$, rolling off to about $44 \mu \mathrm{W}$ at $1 \mathrm{THz}$. The efficiency obtained for this case is $3.32 \%$.

In order to see how the power generation of the resonant-tunneling diode might be improved, it is instructive to re-cast Eq. 3 in a different form. If we assume that the negative differential conductance region is linear such that the current density at the peak current point, $\mathrm{V}_{p}$, is given by $\mathrm{J}_{p}$ and the valley current at $\mathrm{V}_{v}$ is $\mathrm{J}_{v}$, with linear variation between these points, then the conductance per unit area is given by :

$G=\frac{J_{v}-J_{p}}{V_{v}-V_{p}}$

and the rf voltage is given by :

$V_{r f}=\frac{1}{2}\left(V_{v}-V_{p}\right)$.

Substituting these values into Eq. 3 and taking the high-frequency limit, the rf power becomes:

$P_{r f} \approx \frac{J_{p}^{2}\left(\frac{J_{u}}{J_{p}}-1\right)^{2}}{8 \omega^{2} C^{2}} \approx \frac{J_{p}^{2}}{8 \omega^{2} C^{2}}$, 
where the final approximation is obtained by assuming a high peakto-valley ratio. Therefore to optimize the power generation at high frequencies it is necessary to maximize the ratio of peak current to the device capacitance.

One way to increase the device current density is to re-design the structure so that the peak tunneling current is obtained through the second resonant state in the well, rather than the first. The second resonant state tends to be at a higher current density and to have a lower peak-to-valley ratio than the first state. This may be accomplished by constructing the well from lower band-gap material than the emitter [13]. The structure of Fig. 3a differs from that of Fig. 1b only in the well region, which is now $67.4 \AA$ wide and composed of $\operatorname{In}_{.25} \mathrm{Ga}_{.75} \mathrm{As}$, so that the bottom of the quantum well at zero bias is $0.11 \mathrm{eV}$ below the GaAs contact conduction band edge. An additional advantage of the wider well is that the device capacitance is decreased.

The solution of Fig. $3 \mathrm{a}$ is at the peak current point of the I-V curve shown in $3 \mathrm{~b}$. Note that the peak current density has increased from $1.81 \times 10^{5} \mathrm{~A} / \mathrm{cm}^{2}$ for the previous case in Fig. 1a to $2.92 \mathrm{x}$ $10^{5} \mathrm{~A} / \mathrm{cm}^{2}$ for this structure. Since the ground state energy for the well is approximately $70 \mathrm{meV}$ from the bottom, this state is "hidden" and the first calculated peak in the I-V curve of Fig. 3b is due to resonant tunneling through the first excited state. (The doublepeaked density in the well characteristic of resonance through the first excited state is not clearly visible in this figure because waves incident from the right populate the ground state to a higher concentration than the first excited state. However, these waves are not transmitted to the left-hand side of the device and do not contribute to current flow). Small-signal data obtained for this device is shown in Fig. 4a. It is seen that the high-frequency, small-signal response of this device is better than the results for the diode of Fig. 1; the maximum rf power in Fig. $4 \mathrm{~b}$ is $257 \mu \mathrm{W}$, over twice the previous result. The difference at lower frequencies is primarily due to the increase in the peak $\mathrm{V}_{r f}$, which is $22.73 \mathrm{mV}$ for this case. Note that the rf power obtainable at $1 \mathrm{THz}$ is substantially improved also for this structure. The efficiency obtained for this device is $2.96 \%$.

To further improve this device, it is necessary to reduce the device 
capacitance, or to increase the depletion width. Simply widening the AlGaAs barriers to accomplish this would also reduce the tunneling current density. However, by using a stepped-barrier structure [14] or an i-layer [15] on the collector side of the device, the depletion width may be increased without significantly degrading the tunneling current. Fig. 5a shows the dc solution at the peak current point for a structure obtained from that of Fig. 3 by extending the barrier on the collector side by $300 \AA$ at an energy below that of the second resonant state. Note from the density profile in the well that conduction is via the second state. Comparison of the I-V curve in Fig. $5 \mathrm{~b}$ with that of Fig. $3 \mathrm{~b}$ shows that the tunneling current is not affected by the extended barrier. However, the depletion region has been increased to $423 \AA$. The rf voltage range in the negative conductance region has also been expanded, due to the additional voltage drop across the extended barrier.

Fig. 6a presents the small-signal response calculated for this device about the bias point $\mathrm{V}_{d c}=.99 \mathrm{~V}$. The maximum negative conductance is lower than for the previous cases, due to the expanded voltage range. However, the power calculation of Fig. $6 \mathrm{~b}$ shows that $4.11 \mathrm{~mW}$ maximum power is predicted for this device and $2.11 \mathrm{~mW}$ is still obtainable at $1 \mathrm{THz}$. The increased power at high frequencies is due to the reduced capacitance of the device and at lower frequencies to the larger $\mathrm{V}_{r f}$ swing, $90.7 \mathrm{mV}$ for this case. The efficiency obtained is $3.24 \%$.

Table 1 summarizes the rf power results obtained at $10 \mathrm{GHz}$ and $1 \mathrm{THz}$ using the small-signal equivalent circuit. Also shown is the $\mathrm{J}_{p} / \mathrm{C}$ ratio for each device; from $\mathrm{Eq} .7$ the square of this quantity is directly related to the expected power at high frequencies, which is confirmed by the data in Table 1 .

\section{Large-Signal, Quasi-Static Calculations}

In Sec. 2, the rf voltage range was limited so that the device was always operating within the negative differential conductance region 
of the dc I-V curve. Since the I-V curves often fall sharply in this region, this is a severe limitation. It is expected that if this restriction is relaxed, the devices will be capable of generating more power. However, it is also expected that the small-signal equivalent circuit will no longer be applicable. A large-signal, quantum mechanical calculation could in principle be performed, although it would be time consuming and difficult to implement. In this paper, it is simply assumed that under large-signal conditions the device quasi-statically follows the $\mathrm{dc} \mathrm{I}-\mathrm{V}$ curve. A sinusoidal of voltage is assumed across the device, and the resulting current waveforms are Fourier-analyzed to obtain rf power at the fundamental frequency. In addition, a capacitance is added as before to account for displacement current.

Each of the devices analyzed in Sec. 2 was biased near the center of the negative conductance region, and $V_{r f}$ was increased until the maximum power density point was obtained. The area was again obtained by assuming $1 \Omega$ circuit matching. Fig. 7 shows the resulting rf power and device area curves as functions of frequency, at the $V_{r f}$ corresponding to maximum power density. In Fig. 7a, the original device without a deep well or extended barrier generated $4.96 \mathrm{~mW}$ maximum power with $26.2 \%$ efficiency at $10 \mathrm{GHz}$ and $\mathrm{V}_{r f}=.10 \mathrm{~V}$, compared to $97.9 \mu \mathrm{W}$ and $3.32 \%$ efficiency with $V_{r f}=14.0 \mathrm{mV}$ using the small-signal equivalent circuit. Note however that the power falls off faster with frequency than is the case for the small-signal result of Fig. 2b. This is because the device negative conductance is smaller in magnitude for the larger $\mathrm{V}_{r f}$, so that from Eq. 3 the capacitive term becomes dominant at a lower frequency. Comparing the rf power curves at $1 \mathrm{THz}$, it is seen that the power levels for the two cases become comparable. This behavior may also be predicted from Eq. 7, where it is shown that the magnitude of $V_{\tau f}$ becomes less important at very high frequencies.

Fig. 7b shows the quasi-static calculation for the deep-well structure of Fig. 3 where resonance is via the first excited state in the well. For this case $9.76 \mathrm{~mW}$ maximum power and $20.2 \%$ efficiency were obtained at $10 \mathrm{GHz}$ with $\mathrm{V}_{r f}=.14 \mathrm{~V}$, compared to $257 \mu \mathrm{W}$ at $2.96 \%$ efficiency with $\mathrm{V}_{r f}=22.7 \mathrm{mV}$ for the small-signal calculation. Again, the large-signal power results fall off faster with 
frequency than the curve of Fig. $4 \mathrm{~b}$ due to the reduced negative conductance. At $1 \mathrm{THz}$, the large-signal power is less than twice the power obtainable using the small-signal analysis.

Fig. 7c shows the large-signal results for the structure of Fig. 5 , with the $300 \AA$ extended barrier. In this case $129 \mathrm{~mW}$ maximum power at $19.1 \%$ efficiency was obtained at $10 \mathrm{GHz}$ with $\mathrm{V}_{r f}=.51$ $\mathrm{V}$, compared to $4.11 \mathrm{~mW}$ at $3.24 \%$ efficiency with $\mathrm{V}_{r f}=90.7 \mathrm{mV}$ for the small-signal calculation. At $1 \mathrm{THz}$, the power obtained from the quasi-static calculation is again comparable to the small-signal result.

\section{Calculations from Experimental Re- sults and the Thomas-Fermi Model}

Experimental dc I-V curves obtained for devices with a $44 \AA$ In ${ }_{.53} \mathrm{Ga}_{.47}$ As well surrounded by $23.7 \AA$ AlAs barriers are presented in Fig. 8. These devices were grown on $\mathrm{n}^{+} \mathrm{InP}$ substrates, with $\mathrm{In}_{.53} \mathrm{Ga}_{.47} \mathrm{As}$ contact layers Si-doped to $2 \times 10^{18} \mathrm{~cm}^{-3}$ and with $50 \AA$ undoped $\mathrm{In}_{.53} \mathrm{Ga}_{.47} \mathrm{As}$ spacer layers adjacent to the barriers. The peak-to-valley ratios obtained for these devices were 23.9 at room temperature in Fig. $8 \mathrm{a}$ and 51.3 at $77 \mathrm{~K}$ in $8 \mathrm{~b}$, believed to be the highest ever reported for this material system. Shown in Fig. $8 \mathrm{c}$ is a theoretical I-V curve calculated using a Thomas-Fermi selfconsistent method in conjunction with a quantum mechanical eigenstate analysis. In the Thomas-Fermi method, it is assumed that the regions to the left and right of the double-barrier structure are in local equilibrium (constant Fermi level) and electron concentrations are calculated using Fermi-Dirac statistics. Poisson's equation is solved using these concentrations to obtain the conduction band profile. For the calculations presented here, charge in the well was ignored so that constant electric field in this region was assumed. The height of the AlAs barriers was assumed to be $1.2 \mathrm{eV}$. Fig. $8 \mathrm{~d}$ shows a dc solution at the peak current point of $8 \mathrm{c}$ using this method. In this figure the electron concentration calculated from the ThomasFermi method outside the double-barrier structure is shown as a 
solid curve, while the electron concentration in the structure calculated from the quantum mechanical eigenstate analysis is shown as a dashed curve. It is seen that this method results in a discontinuity in concentration profiles at the boundaries.

From Fig. 8c, it is seen that the theoretical peak current density is $0.928 \times 10^{4} \mathrm{~A} / \mathrm{cm}^{2}$, whereas the experimental peak current density from Fig. $8 \mathrm{a}$ is $1.5 \times 10^{4} \mathrm{~A} / \mathrm{cm}^{2}$. The theoretical peak current density is lower due to the assumption in the program that the effective mass assumes a higher value of $.15 \mathrm{~m}_{0}$ within the entire $23.7 \AA$ AlAs barrier region, compared to $.042 \mathrm{~m}_{0}$ in the contacts and well. In fact, electrons entering the barrier regions require a finite distance before this effective mass value can be exhibited. Reducing the effective mass discontinuity results in a higher calculated peak current density. However, in practice it is difficult to determine an appropriate effective mass vs. distance profile to use.

Fig. 9a shows the large-signal rf power predicted based on the theoretical I-V curve of Fig. $8 \mathrm{c}$, and $9 \mathrm{~b}$ shows the large-signal calculation using the experimental I-V curve of Fig. 8a. In these calculations, the depletion width was estimated based on a calculation of the incremental device capacitance using the Thomas-Fermi method. For this case, $D=267.2 \AA$ was used. As expected, the high-frequency performance of this device is severely degraded due to the low value of peak current density. Therefore an important issue for these devices is to find methods of improving the current density without significantly compromising the peak-to-valley ratio.

In order to increase the current density for this device while maintaining a reasonable peak-to-valley ratio, the barrier heights may be lowered. A series of I-V curve calculations was carried out with the barrier heights as parameters. It was found that an optimum configuration (using the $\mathrm{J}_{p} / \mathrm{C}$ criterion ) was a slightly asymmetric structure with one barrier height equal to $.53 \mathrm{eV}$ and the other $.6 \mathrm{eV}$. These barrier heights may be realized by forming the barriers from $\mathrm{In}_{x} \mathrm{Al}_{1-x} \mathrm{As}$ and adjusting the In concentration. Fig. 10a shows a Thomas-Fermi self-consistent solution at the peak current point for the improved structure. The theoretical I-V curve in $10 \mathrm{~b}$ shows that peak current densities of $8.6 \times 10^{5} \mathrm{~A} / \mathrm{cm}^{2}$ are predicted, with a peakto-valley ratio of 18.2 . The peak current is high since the barriers 
for this structure are very thin. The quasi-static power calculation for this device is shown in Fig. 10c. The performance is significantly improved due to the increased current density.

Table 2 summarizes the results obtained for $\mathrm{rf}$ power using the quasi-static analysis. The highest power at high frequencies is obtained for the asymmetric-barrier structure with $50 \AA$ spacer layers of Fig. 10, for which the peak current density is also the highest. The second highest power is obtained for the stepped-barrier structure of Fig. 5. Note in all cases that the $\mathrm{J}_{p} / \mathrm{C}$ ratio is a good indication of relative power output at high frequencies.

\section{Conclusions}

It has been shown that an important figure of merit for high-frequency power generation by resonant-tunneling diodes is the ratio of peak current density to device capacitance. Methods for increasing peak current and reducing capacitance have been proposed, including second-level resonance, barrier height optimization and incorporation of an extended barrier or i-layer. Several milliwatts of power are predicted theoretically for optimized devices up to $1 \mathrm{THz}$.

\section{Acknowledgments}

This work was supported by the US Army Research Office under the URI program, contract no. DAAL03-87-K-0007. 
Table 1: Summary of rf power obtained at $10 \mathrm{GHz}$ and $1 \mathrm{THz}$ from the smali-signal analysis method, together with $\mathrm{J}_{p} / \mathrm{C}$ ratio.

\begin{tabular}{|c|c|c|c|}
\hline Structure(Fig.No.) & $\mathbf{P}(\mathbf{1 0 G H z})(\mathrm{mW})$ & $\mathbf{P}(\mathbf{1 T H z})(\mathrm{mW})$ & $\mathbf{J p} / \mathbf{C}\left(10^{11} \mathrm{~V} / \mathrm{sec}\right)$ \\
\hline \hline 1 & .0979 & .044 & 1.72 \\
\hline 3 & .257 & .1789 & 3.39 \\
\hline 5 & 4.11 & 2.11 & 11.4 \\
\hline
\end{tabular}

Table 2: Summary of rf power obtained at $10 \mathrm{GHz}$ and $1 \mathrm{THz}$ from the quasi-static analysis, together with $\mathrm{J}_{p} / \mathrm{C}$ ratio. Results from experimental curve labeled $\mathrm{E}$.

\begin{tabular}{|c|c|c|c|}
\hline Structure(Fig.No.) & $\mathbf{P}(\mathbf{1 0 G H z})(\mathrm{mW})$ & $\mathbf{P}(1 \mathbf{T H z})(\mathrm{mW})$ & $\mathbf{J p} / \mathrm{C}\left(10^{11} \mathrm{~V} / \mathrm{sec}\right)$ \\
\hline \hline 1 & 4.96 & .0705 & 1.72 \\
\hline 3 & 9.76 & .265 & 3.39 \\
\hline 5 & 129. & 2.53 & 11.4 \\
\hline 8 & 5.64 & .0007 & .233 \\
& $13 .(\mathrm{E})$ & $.00169(\mathrm{E})$ & $.315(\mathrm{E})$ \\
\hline 10 & 33.8 & 6.22 & 20.9 \\
\hline
\end{tabular}



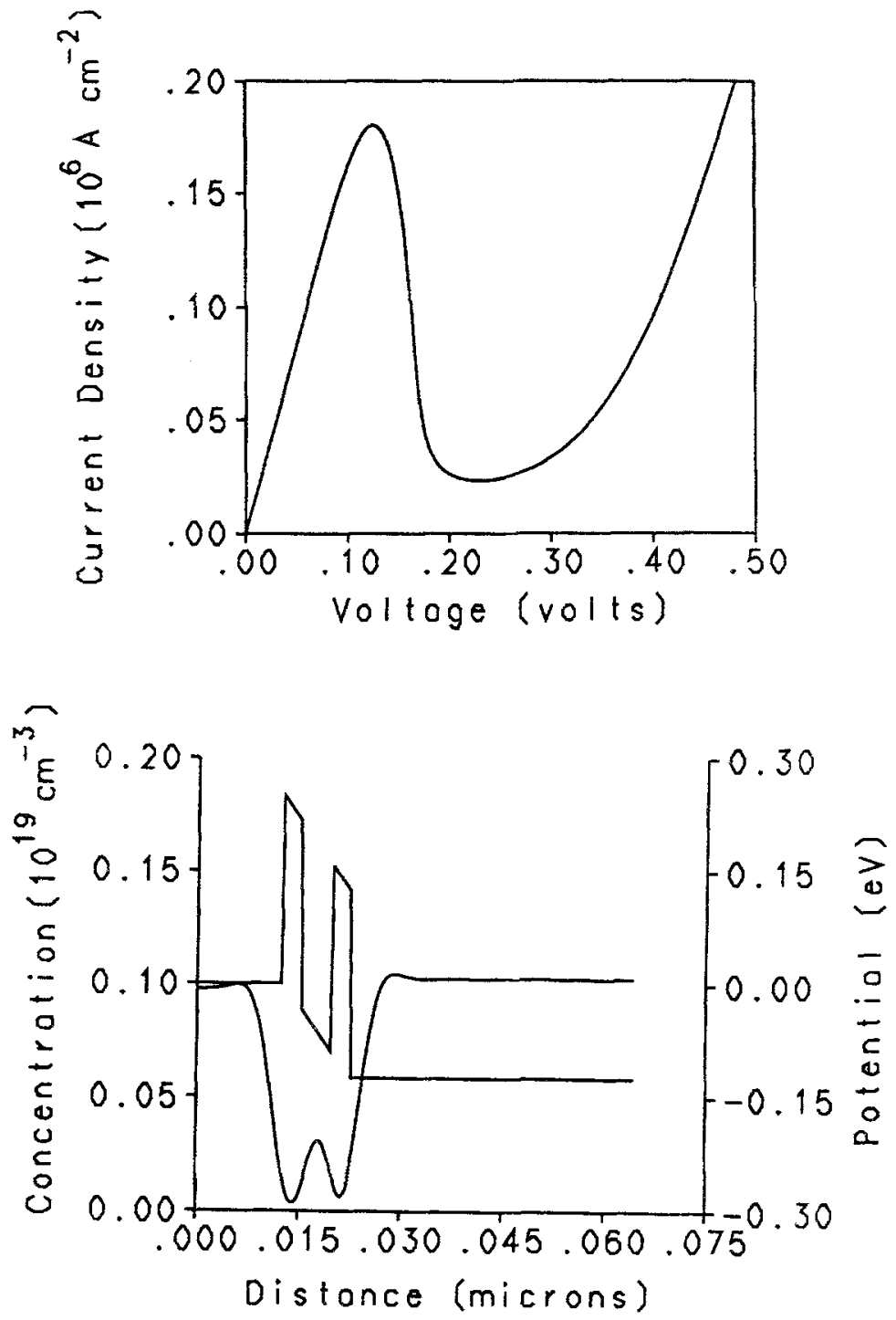

Figure 1: (a) DC I-V curve calculated for GaAs - $\mathrm{Al}_{3} \mathrm{Ga}_{.7} \mathrm{As}-\mathrm{GaAs}$ device with $28 \AA$ barriers and $44.9 \AA$ well and (b) solution for $\mathrm{V}_{d c}=.125 \mathrm{~V}$, at the peak current point. 

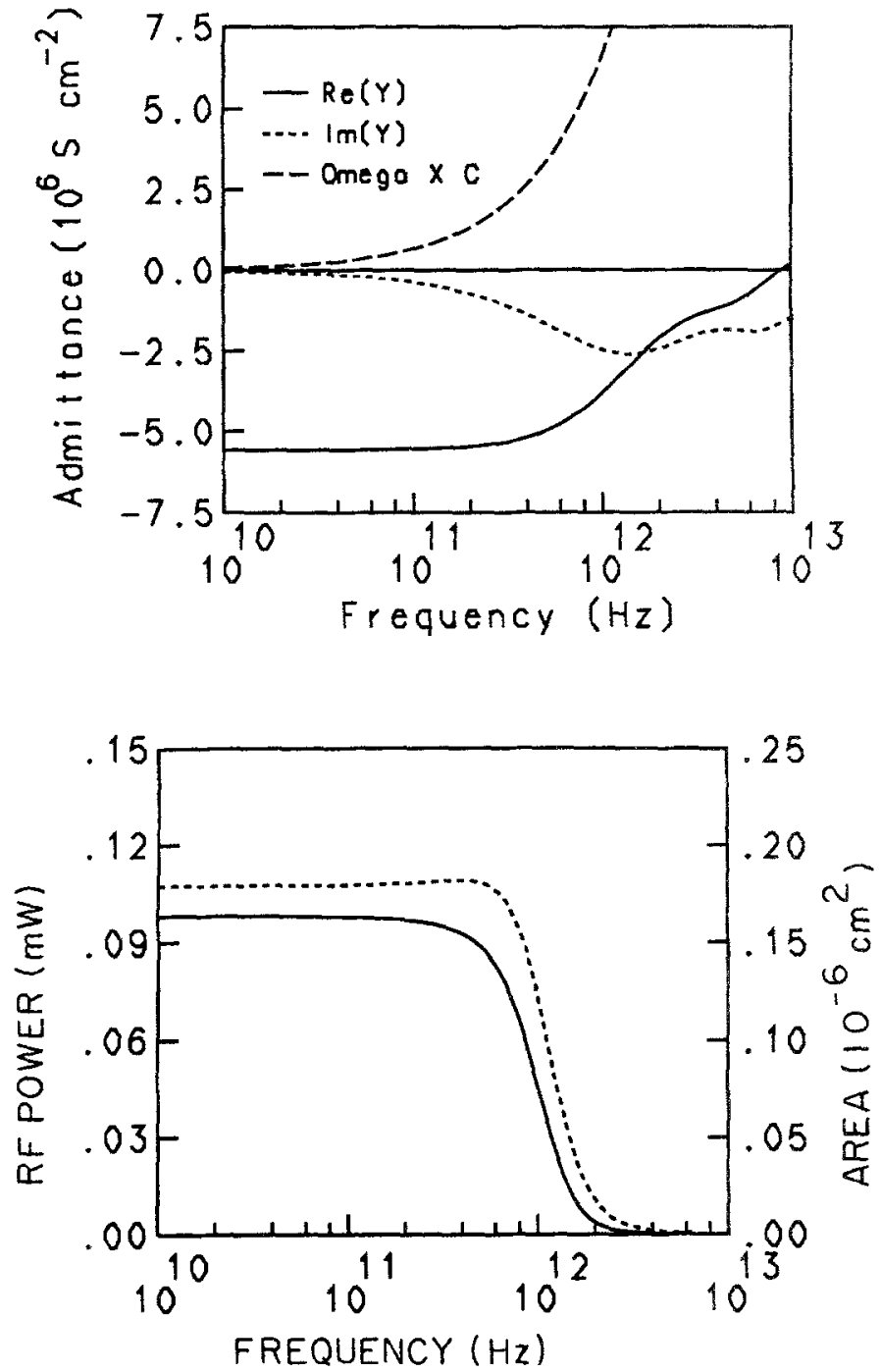

Figure 2: (a) Small-signal admittance for the device of Fig. 1 with $V_{d c}=$ $.1615 \mathrm{~V}$, and (b) estimated if power (solid curve) for the case of $1 \Omega$ matching $\left(V_{r f}=14.0 \mathrm{mV}\right)$. 

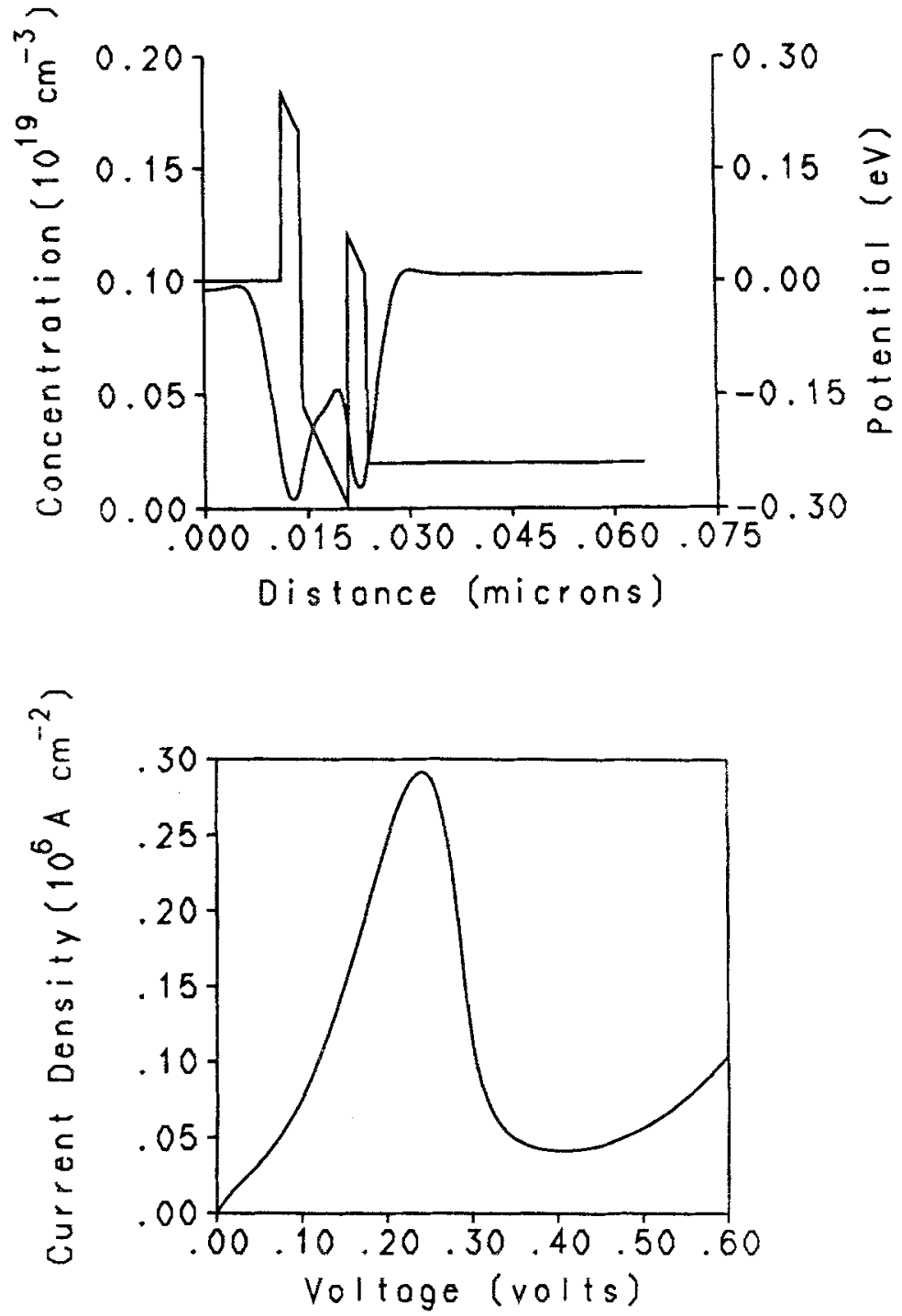

Figure 3: (a) DC solution at $V_{d c}=.24 \mathrm{~V}$ (peak current point) for a GaAs$\mathrm{Al}_{3} \mathrm{Ga}_{7}$ As - In ${ }_{25} \mathrm{Ga}_{.75}$ As device with $28 \AA$ barriers and $67.4 \AA$ well and (b) dc I-V curve calculated for this structure. 

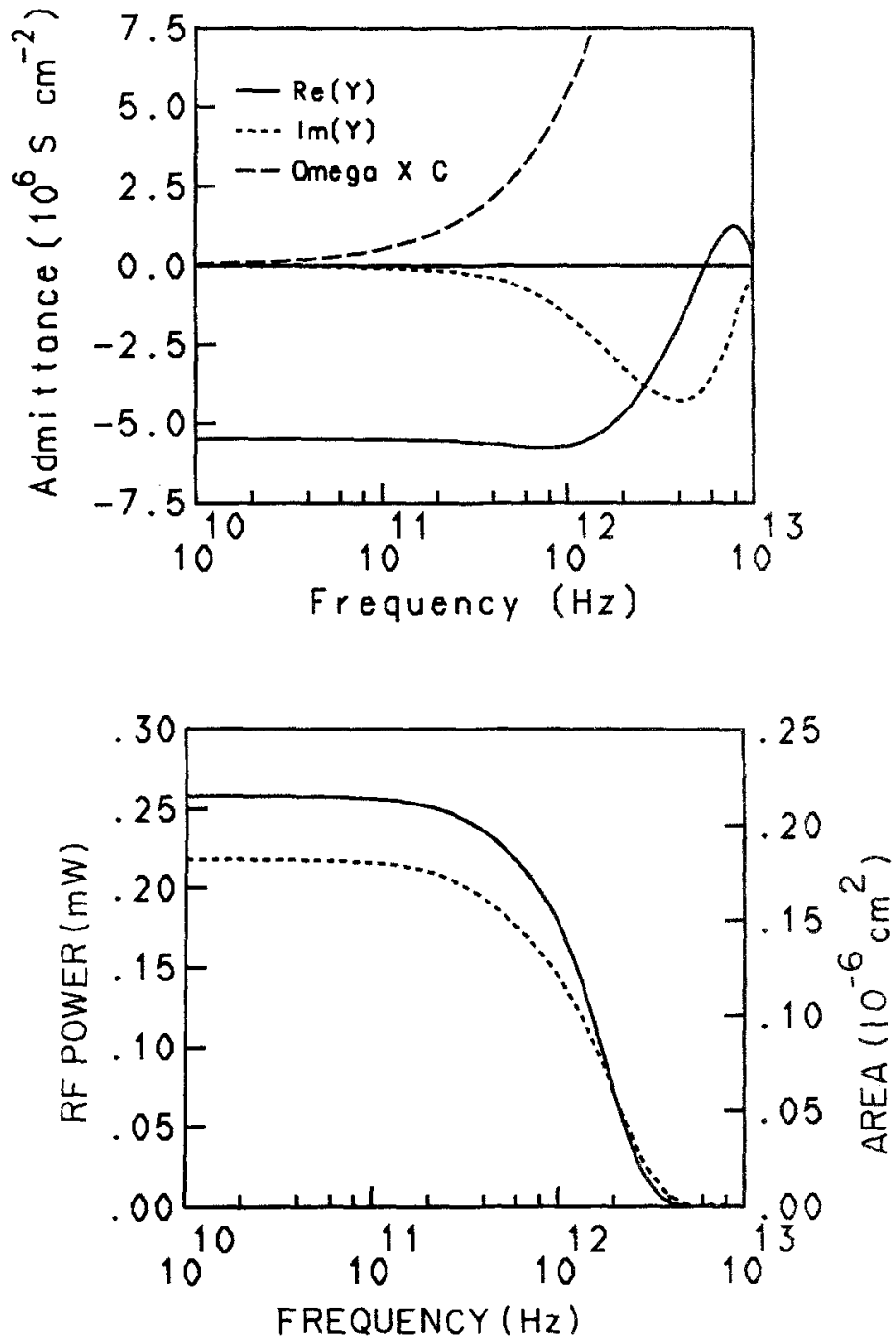

Figure 4: (a) Small-signal admittance for the device of Fig. 3 with $V_{d c}=$ $.2877 \mathrm{~V}$, and (b) estimated rf power (solid curve) for the case of $1 \Omega$ matching $\left(V_{r f}=22.73 \mathrm{mV}\right)$. 

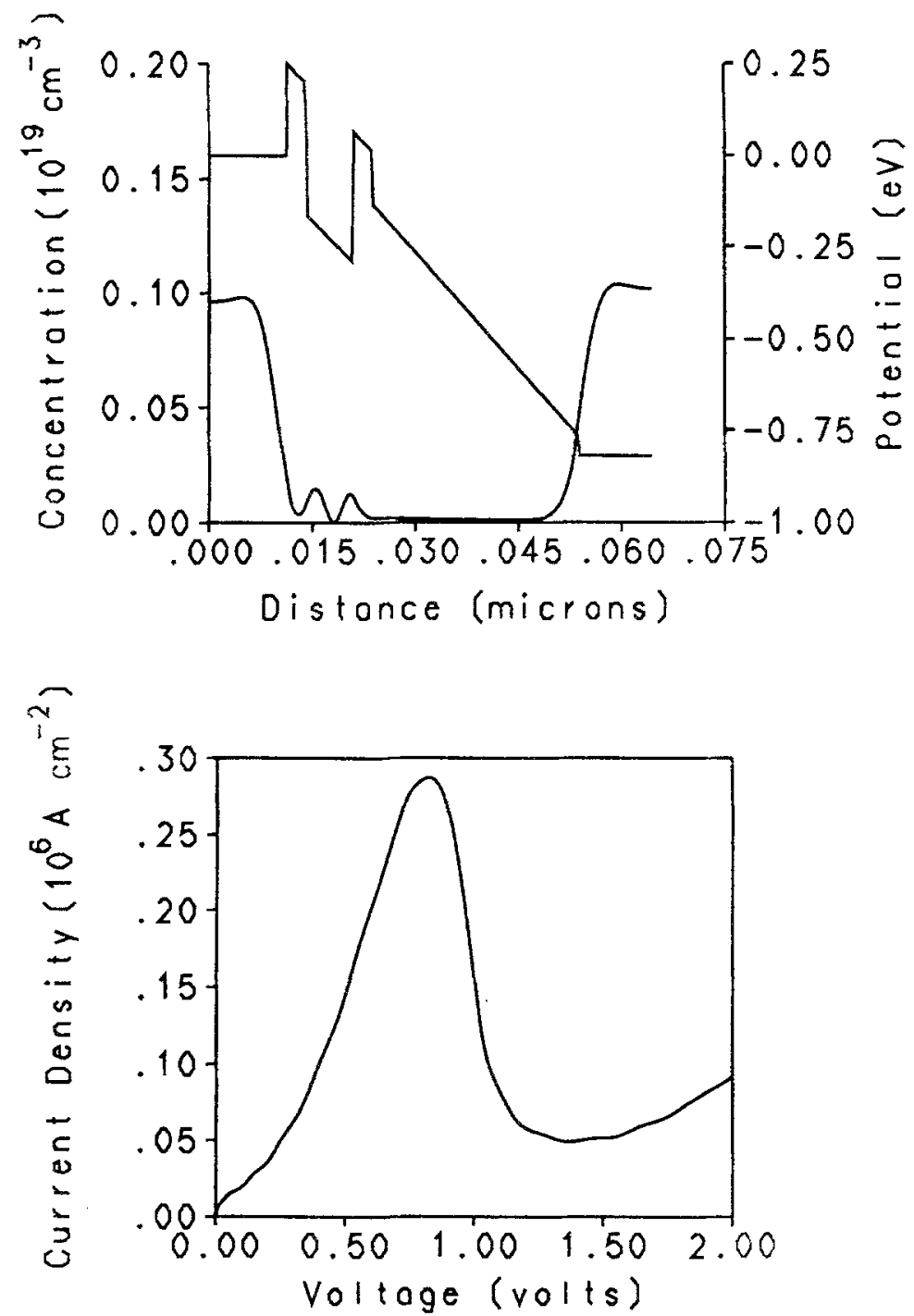

Figure 5: (a) DC solution at $V_{d c}=.82 \mathrm{~V}$ (peak current poirt) for a GaAs$\mathrm{Al}_{.3} \mathrm{Ga}_{.7} \mathrm{As}$ - In ${ }_{.25} \mathrm{Ga}_{.75} \mathrm{As}$ device with $67.4 \AA$ well, $28 \AA$ barrier on emitter side and stepped barrier on collector side and (b) dc I-V curve calcuiated for this structure. 

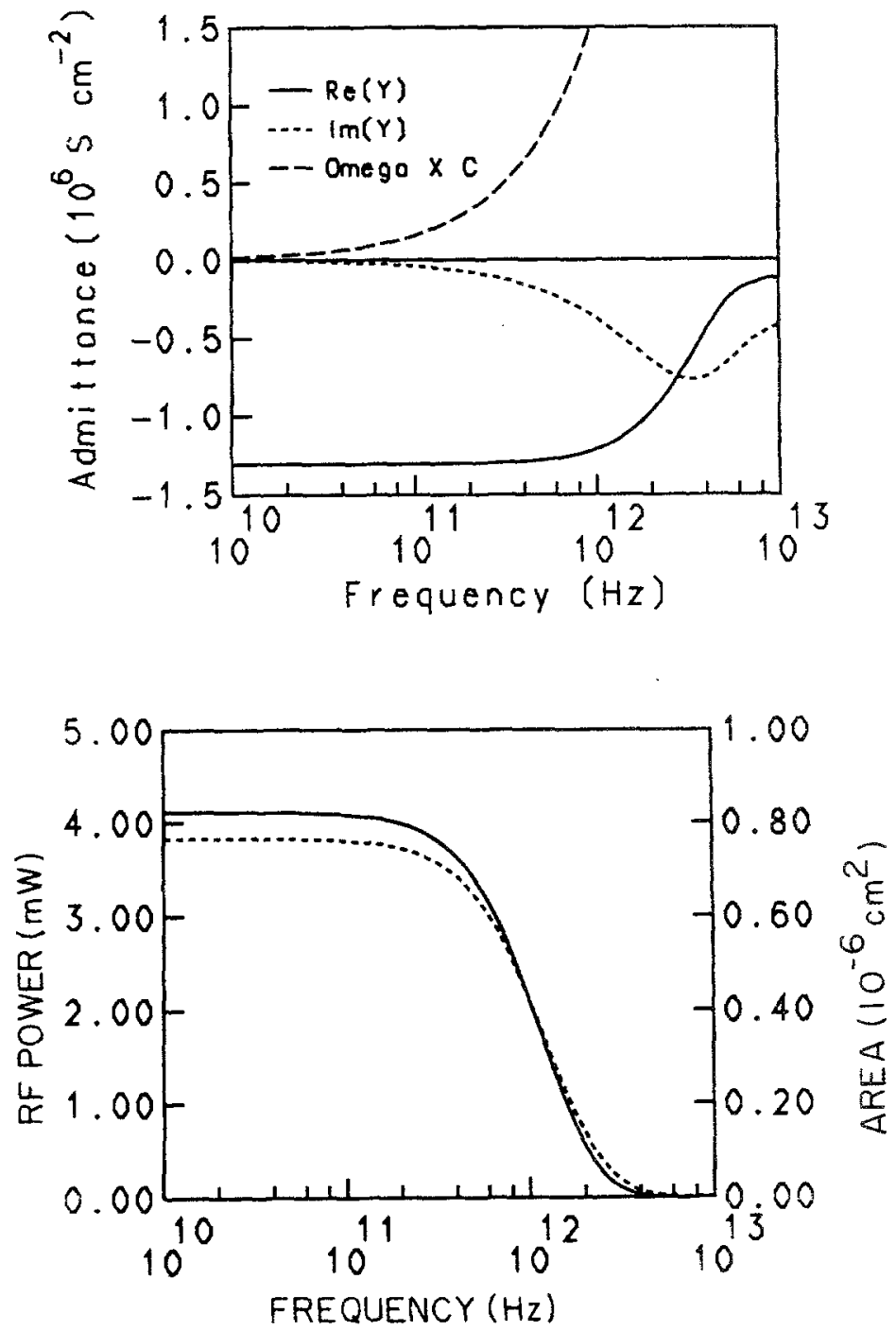

Figure 6: (a) Small-signal admittance for the device of Fig. 5 with $V_{d c}=$ $.99 \mathrm{~V}$, and (b) estimated of power (solid curve) for the case of $1 \Omega$ matching $\left(V_{r f}=90.7 \mathrm{mV}\right)$. 


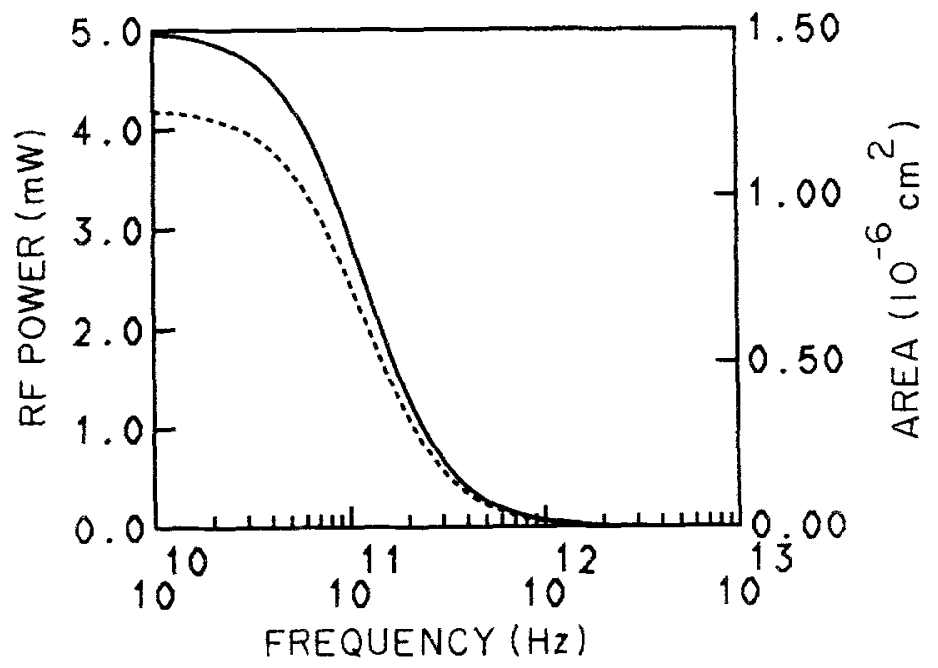

Fig. 7a

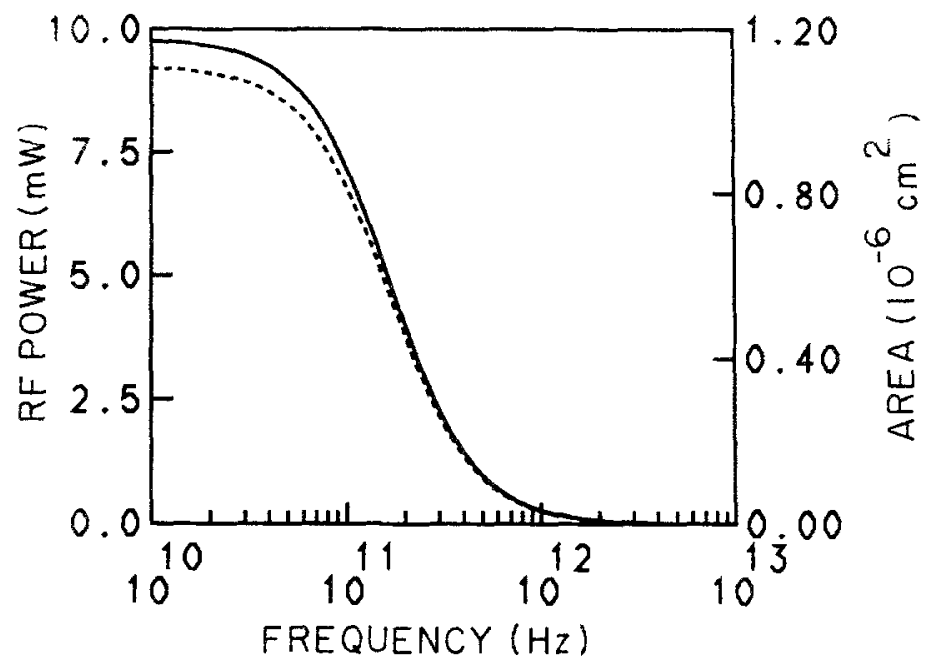

Fig. $7 b$ 


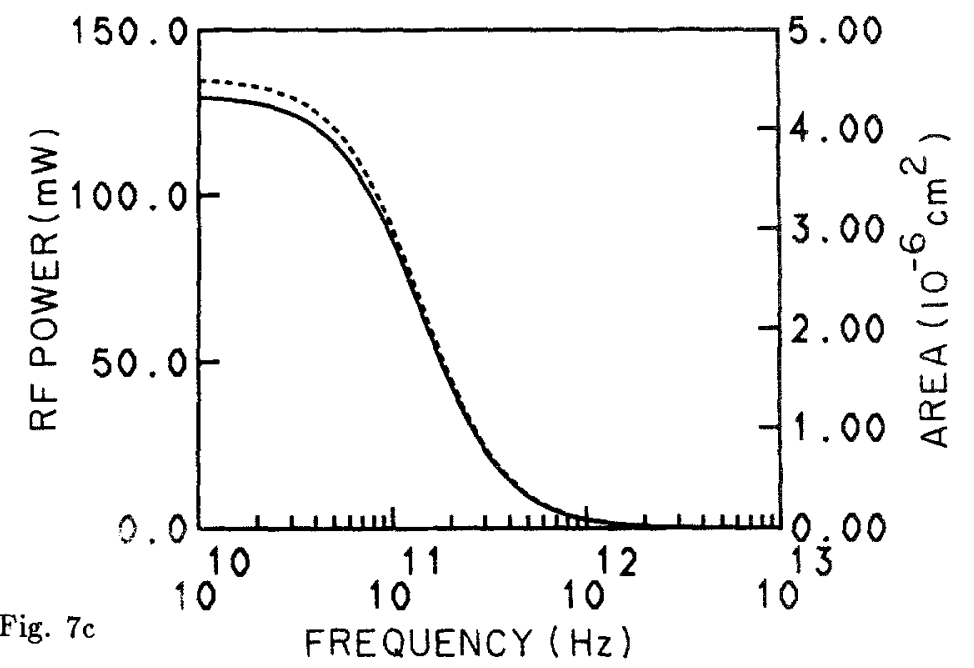

Figure 7: Quasi-static calculation of power (solid) and device area (dashed) for (a) the structure of Fig. I with $V_{d c}=.177 \mathrm{~V}, V_{r f}=.10 \mathrm{~V}$, (b) structure of Fig 3 with $V_{d c}=.3215 \mathrm{~V}, V_{r f}=.14 \mathrm{~V}$, and (c) structure of Fig. jo with $\mathrm{V}_{d c}=1.092 \mathrm{~V}, \mathrm{~V}_{r f}=.51 \mathrm{~V}$.

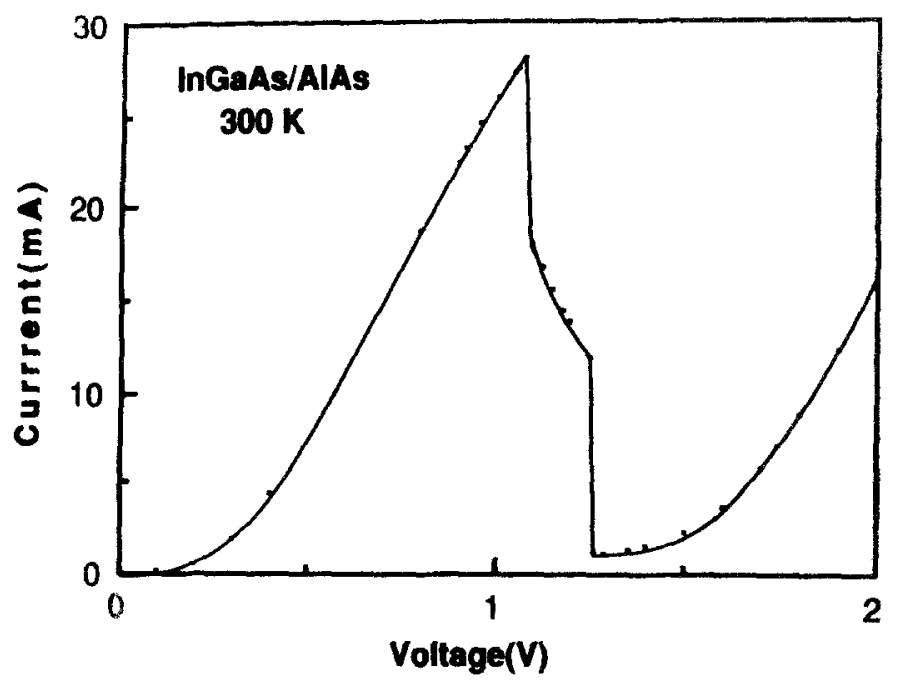

Fig. $8 \mathrm{a}$ 


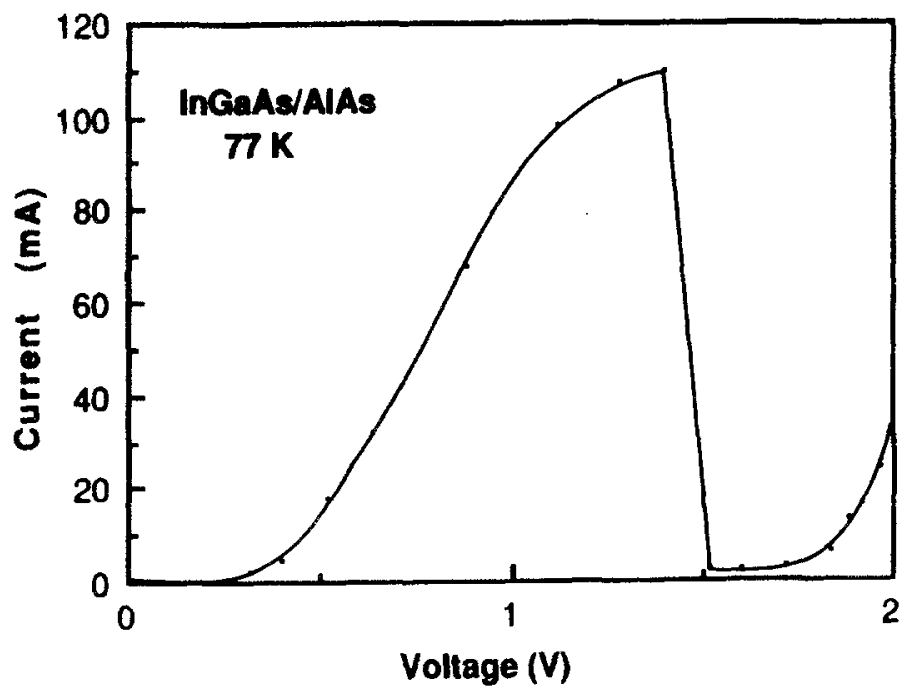

Fig. $8 b$

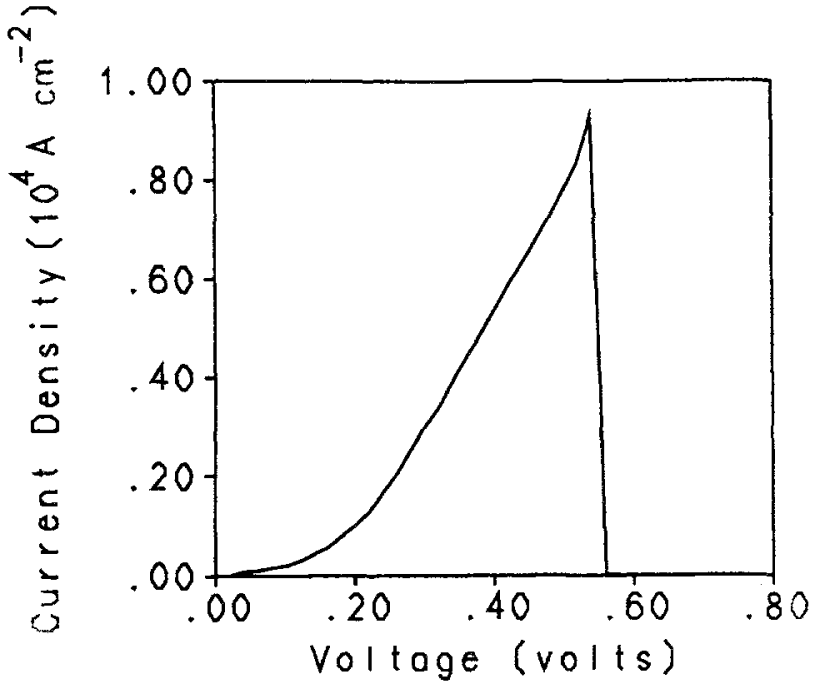

Fig. $8 \mathrm{c}$ 


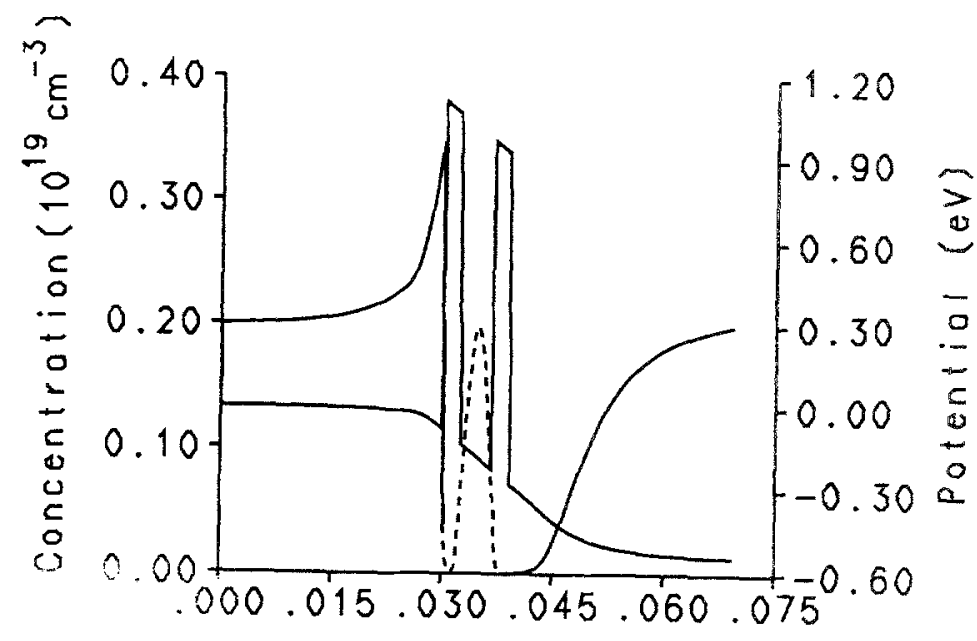

Fig. $8 \mathrm{~d}$

$$
\text { Distonce (microns) }
$$

Figure 8: Experimental dc I-V curves for $44 \AA$ In $_{.53} \mathrm{Ga}_{.47}$ As well $-23.7 \AA$ AlAs barrier structure (a) at room temperature with peak-to-valley ratio of 23.9 , device diameter $=16 \mu \mathrm{m}$, (b) at $77 \mathrm{~K}$ with peak-to-valley ratio of 51.3 , device diameter $=28 \mu \mathrm{m}$, (c) theoretical I-V curve for this structure using ThomasFermi method with peak-to-valley ratio of 3119 , and (d) dc solution at $V_{d c}$ $=.54 \mathrm{~V}$, the peak-current point.

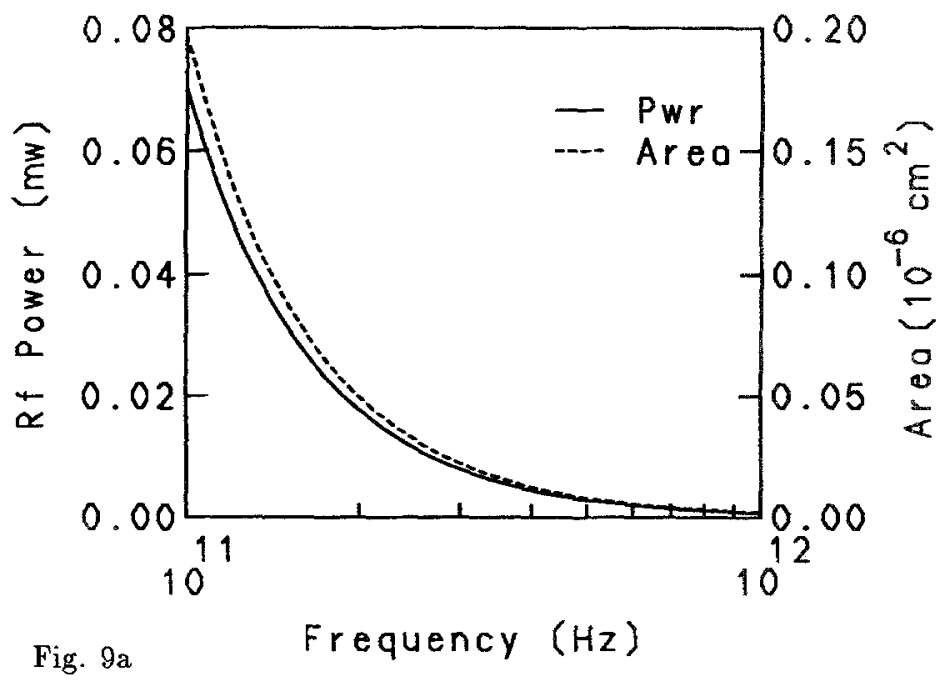




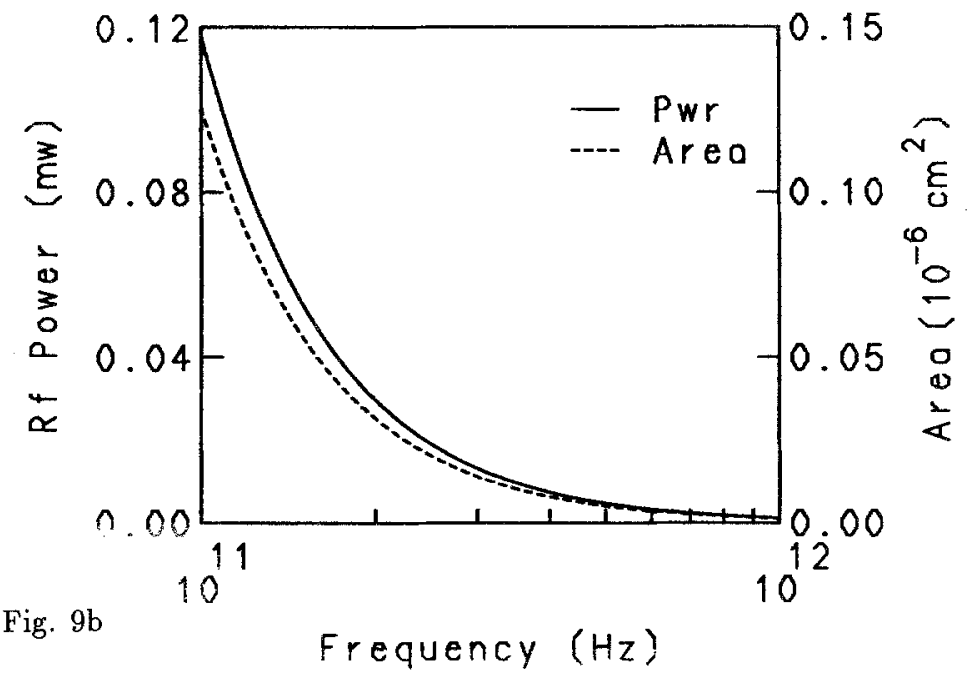

Figure 9: Quasi-static calculation of power (solid) and device area (dashed) for the device of Fig. 8 using (a) theoretical I-V curve of $8 \mathrm{c}$ with $V_{d c}=.56$ $\mathrm{V}, \mathrm{V}_{\tau f}=.24 \mathrm{~V}$ and (b) experimental I- $\mathrm{V}$ curve of $8 \mathrm{a}$ with $\mathrm{V}_{d c}=1.17 \mathrm{~V}, \mathrm{~V}_{\tau f}$ $=.44 \mathrm{~V}$.

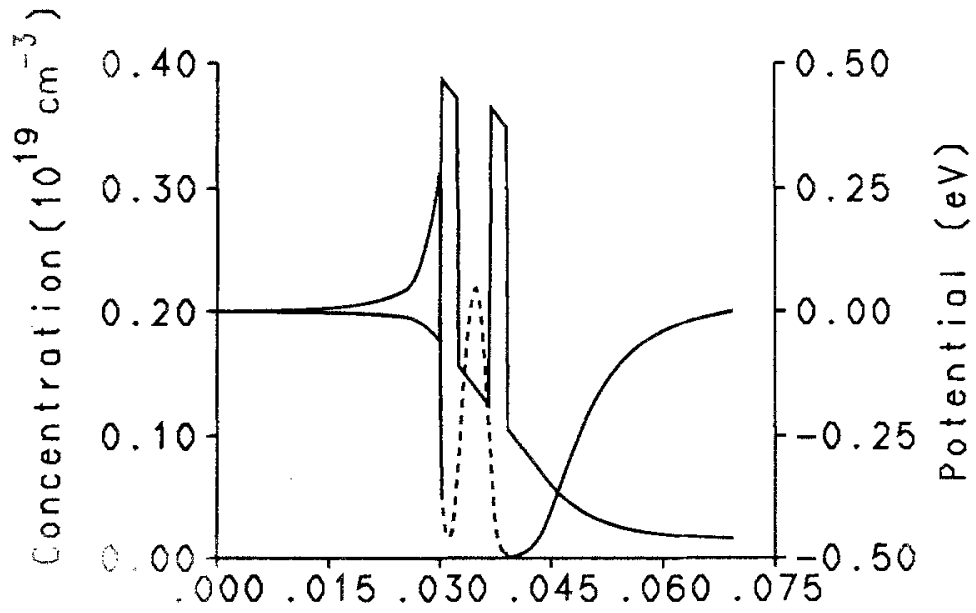

Fig. 10a Distance (microns) 

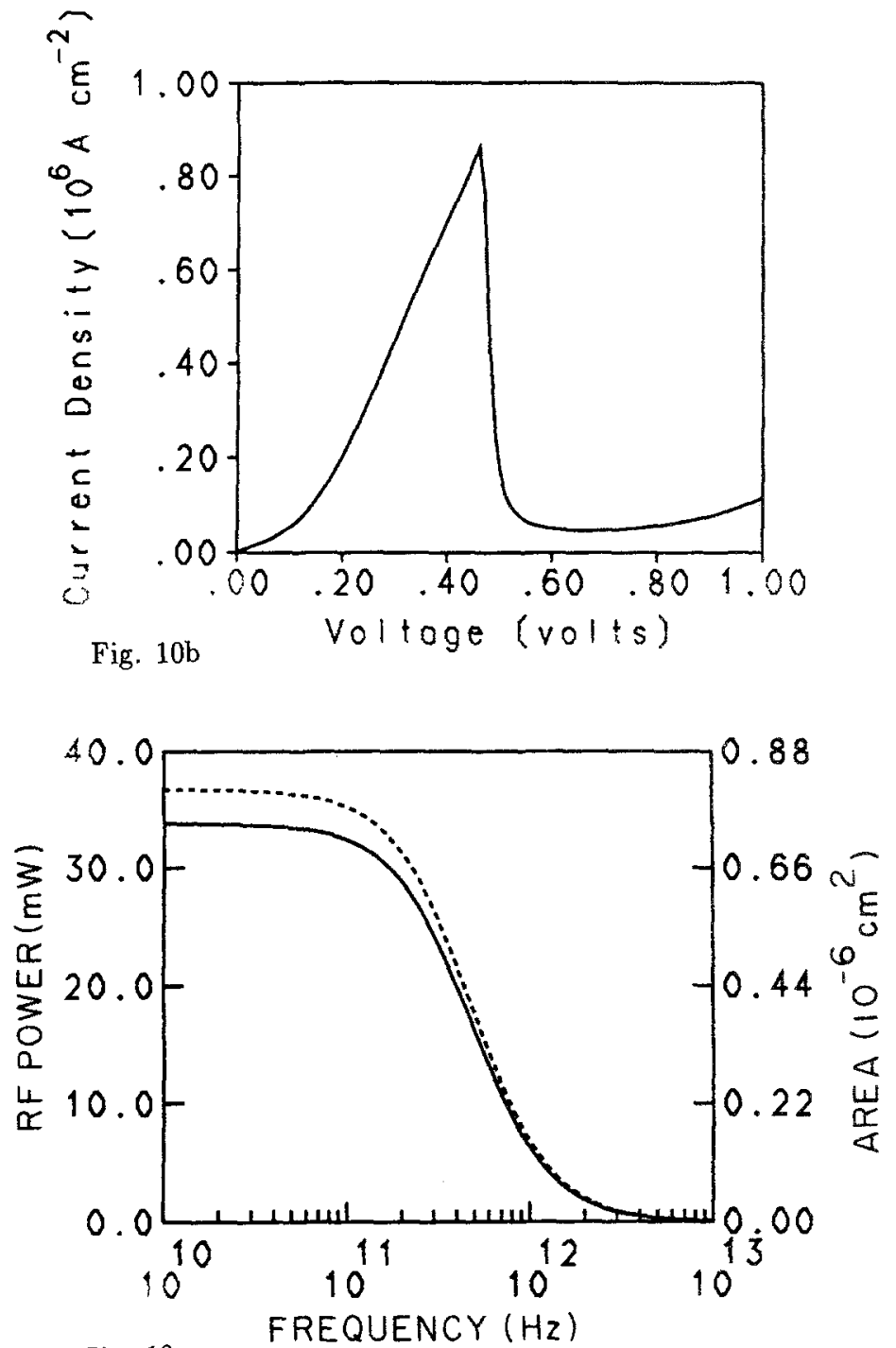

Fig. 10c

Figure 10: (a) Thomas-Fermi solution at peak current for $44 \AA \operatorname{In}_{.53} \mathrm{Ga}_{.47} \mathrm{As}$ well $-23.7 \mathrm{~A}$ barrier structure with $V_{B 1}=.53 \mathrm{eV}$. $V_{B_{2}}=.6 \mathrm{eV}$. (b) calculated I- $V$ curve for this structure, and (c) quasi-static calculation of rf power (solid) and device area (dashed) for $\mathrm{V}_{d c}=.5633 \mathrm{~V}, \mathrm{~V}_{r f}=.26 \mathrm{~V}$. 


\section{References}

[1] B. Jogai, K. L. Wang and K. W. Brown, "Frequency and Power Limit of Quantum Well Oscillators", Appl. Phys. Lett. 48 (15), pp. 1003-1005, 14 April 1986.

[2] D. D. Coon and H. C. Liu, "Frequency Limit of Double Barrier Resonant Tunneling Oscillators", Appl. Phys. Lett. 49 (2), pp. 94-96, 14 July 1986.

[3] T. C. L. G. Sollner, E. R. Brown, W. D. Goodhue and H. Q. Le, "Observation of Millimeter-Wave Oscillations from Resonant Tunneling Diodes and Some Theoretical Considerations of Ultimate Frequency Limits", Appl. Phys. Lett. 50 (6), pp. 332-334, 9 February 1987.

[4] D. S. Pan and C. C. Meng, "On the Mechanism and Frequency Limit of Double-Barrier Quantum-Well Structures", Appl. Phys. Lett. 61 (5), pp. 2082-2084, 1 March 1987.

[5] W. R. Frensley, "Wigner-Function Model of a ResonantTunneling Semiconductor Device", Phys. Rev. B, vol. 36, no. 3, pp. 1570-1580, 15 July 1987.

[6] W. R. Frensley, "Quantum Transport Calculation of the SmallSignal Response of a Resonant Tunneling Diode", Appl. Phys. Lett. 51 (6), pp. 448-450, 10 August 1987.

[7] N. C. Kluksdahl, A. M. Kriman, D. K. Ferry and C. Ringhofer, "Transient Switching Behavior of the Resonant-Tunneling Diode", IEEE Elec. Dev. Lett., vol. 9, no. 9, pp. 457-459, September 1988.

[8] R. K. Mains and G. I. Haddad, "Wigner Function Modeling of Resonant Tunneling Diodes with High Peak-to-Valley Ratios", J. Appl. Phys. 64 (10), pp. 5041-5044, 15 November 1988.

[9] R. K. Mains and G. I. Haddad, "Time-Dependent Modeling of Resonant-Tunneling Diodes from Direct Solution of the 
Schrödinger Equation", J. Appl. Phys. 64 (7), pp. 3564-3569, 1 October 1988.

[10] E. R. Brown, T. C. L. G. Sollner, W. D. Goodhue and C. D. Parker, "Millimeter-Band Oscillations Based on Resonant Tunneling in a Double-Barrier Diode at Room Temperature", Appl. Phys. Lett. 50 (2), pp. 83-85, 12 January 1987.

[11] E. R. Brown, T. C. L. G. Sollner, W. D. Goodhue and C. L. Chen, "High-Speed Resonant-Tunneling Diodes", Proc. of SPIE, Quantum Well and Superlattice Physics II, published by SPIE, Bellingham, Washington, pp. 2-13, Newport Beach, California, 17-18 March 1988.

[12] T. C. L. G. Sollner, W. D. Goodhue, P. E. Tannenwald, C. D. Parker and D. D. Peck, "Resonant Tunneling Through Quantum Wells at Frequencies up to $2.5 \mathrm{THz}$ ", Appl. Phys. Lett. 43 (6), pp. 588-590, 15 September 1983.

[13] M. A. Reed and J. W. Lee, "Resonant Tunneling in a GaAs/AlGaAs Barrier/InGaAs Quantum Well Heterostructure", Appl. Phys. Lett. 50 (13), pp. 845-847, 30 March 1987.

[14] G. I. Haddad, R. K. Mains, U. K. Reddy and J. R. East, "A Proposed Narrow-Band-Gap Base Transistor Structure", presented at Fourth Inter. Conf. on Superlattices, Microstructures and Microdevices, Trieste, Italy, August 1988.

[15] V. P. Kesan, D. P. Neikirk, B. G. Streetman, and P. A. Blakey, "A New Transit-Time Device Using Quantum-Well Injection", IEEE Elec. Dev. Lett., vol. EDL-8, no. 4, pp.129-131, April 1987. 